\title{
Idiosyncrasies of COVID-19; A Review
}

\author{
Nzube Ekpunobi ${ }^{*}$, Irene Markjonathan ${ }^{1}$, Oluwadamilola Olanrewaju ${ }^{1}$, David Olanihun ${ }^{1}$ \\ 1. Department of Microbiology, Faculty of Science, University of Lagos, Akoka, Nigeria
}

\section{ABSTRACT}

The Coronavirus disease 2019, identified by Chinese researchers to be the caused by a novel enveloped betacoronavirus, Severe Acute Respiratory Syndrome Coronavirus- 2 which was first isolated in Wuhan, China has been declared a global pandemic by the world health organization. The virus has several structural proteins that contributed to its pathogenesis such as spikes, membrane, envelop and nucleocapsid protein facilitating its attachment, entry and cell-to-cell transmission. The virus is readily transmitted through human-to-human contact and there is presently no approved vaccine for its prevention. This study was carried out to review the epidemiology of Severe Acute Respiratory Syndrome Coronavirus- 2, its host and reservoir, pathogenesis, transmission, clinical manifestation and potential treatment options for the infection.

Keywords: Coronavirus disease 2019, Severe Acute Respiratory Syndrome Coronavirus- 2, Structural proteins, Pathogenesis, Epidemiology, Transmission, Hosts and Reservoir

Received: 2019/10/29; $\quad$ Accepted: 2020/03/21; Published Online: 2020/05/12

\begin{tabular}{|c|c|}
\hline Corresponding Information: & $\begin{array}{l}\text { Ebrahim Babapour, 2- Department of Microbiology, Faculty of Science, University of Lagos, Akoka, Nigeria } \\
\text { Email: nzubefavour34@gmail.com }\end{array}$ \\
\hline (c) $\underset{\mathrm{BY}}{(1)}(\mathrm{NC}$ & $\begin{array}{l}\text { This is an original open-access article distributed under the terms of the Creative Commons Attribution-noncommercial } 4.0 \text { International License which } \\
\text { distribution of the material just in noncommercial usages with proper citation. }\end{array}$ \\
\hline
\end{tabular}

Use your device to scan and read the article online

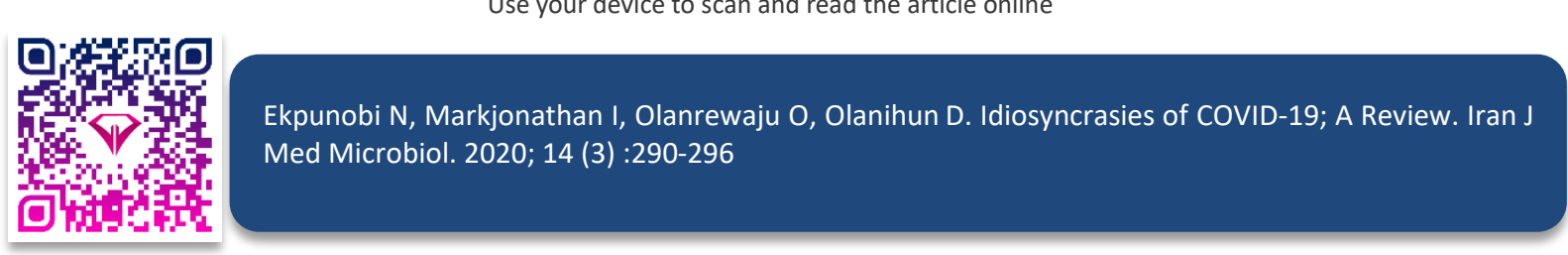

Download citation: BibTeX | RIS | EndNote | Medlars | ProCite | Reference Manager | RefWorks

Send citation to: $\otimes_{\text {Mendeley }} \mathbf{z}$ zotero $\mathbb{H}_{\text {RefWorks }}$

\section{Introduction}

Coronaviruses are enveloped single stranded positive sense RNA viruses that are zoonotic in nature (1). The coronaviruses genome is said to be the largest known RNA virus genome with a genome size approximately 26-32 kilobases (2).

The coronavirus belongs to the order Nidovirales under the family coronaviridae. The coronavirinae which is one of the subfamilies of the coronaviridae family is further subdivided into four groups; the Alpha, Beta, Gamma and Delta coronaviruses. All coronaviruses have 16 nonstructural proteins (nsp1-nsp16) and 4 structural proteins (3) except the betacoronaviruses with 5 structural proteins (4). The four main structural proteins are spike $(S)$, membrane $(M)$, envelope $(E)$, and nucleocapsid $(N)$ proteins $(3,5)$.
The coronavirus disease 2019 (COVID-19) has been identified to be caused by a novel enveloped RNA betacoronavirus belonging to the orthocoronavirinae subfamily $(6,7)$. As a result of the phylogenetic similarity between the causal pathogen of COVID-19 and SARS-CoV, the novel virus is currently called Severe Acute Respiratory Syndrome Coronavirus-2(SARS-CoV-2). This SARS-CoV-2 is a strain of severe acute respiratory syndrome-related coronavirus (SARSr-CoV) (8). The virus genome consists of six open reading frames (ORFs) that are common to coronaviruses and its genes shared more than $80 \%$ nucleotide sequence identity to SARS-CoV. The disease presents with complications such as severe pneumonia, respiratory failure, acute respiratory distress syndrome (ARDs) and cardiac injury including fatal outcomes $(9,10)$. 
This study was carried out to review the epidemiology of SARS-CoV-2, its host and reservoir, pathogenesis, transmission, clinical manifestation and potential treatment options for the infection.

\section{Materials and Methods}

\section{Strategies Used in Searching for Literatures}

Literature search for the review was performed in "Science direct", "Google scholar", "PubMed" and also in "MDPI", THE LANCET, "Bioxriv", "JAMA" and "NATURE" journals using the search terms; COVID-19, SARS-CoV-2 and Coronavirus to find articles published before April 13, 2020. Also, some published articles by the WHO and Center for disease prevention and control were included in the analysis. Studies were excluded if data was old and were not pertinent to the aim of the study.

\section{Identification of Relevant Studies}

Identification of studies meeting the inclusion criteria was done by two review authors, who screened the titles and abstracts of all retrieved articles. After the selected articles were read, a conclusive decision was made for each study. The inclusion criteria include studies based on experimental data, studies discussing either clinical manifestation, treatment options of SARS-CoV-2 or both, studies based on transmission and host reservoir of SARS-CoV-2 and studies containing information on epidemiology, pathogenesis and symptoms of SARSCoV-2

A total of 56 articles were screened based on the inclusion criteria and 43 studies were selected and used for the review.

\section{Extraction of Data from Selected Studies}

Data was extracted from all selected articles independently by all four reviewers based on the main research objectives; epidemiology, host and reservoir, pathogenesis, transmission, clinical manifestation and potential treatment options for COVID-19.

All findings and statements regarding the outbreak in this study are based on published information as listed in references.

\section{Results and Discussion}

\section{Epidemiology}

Early cases of COVID-19 were found to have contact history with the original seafood market, however, human-to-human transmission has been identified as disease progresses (11).The first four cases reported, all linked to the Huanan (Southern China) seafood wholesale market, were identified by local hospitals using surveillance mechanism for "pneumonia of unknown etiology" on December 29, 2019 (12). Laboratory-confirmed 2019-nCoV (COVID-19) cases were identified in 41 admitted hospital patients in Wuhan, China as at January 2, 2020. Most of these patients were reported to have had recent exposure to the Huanan seafood wholesale market, while, half of these patients were reported to have underlying diseases such as diabetes, hypertension and cardiovascular diseases (9).

On January 7, 2020, a novel member of enveloped RNA coronavirus was identified in samples of bronchoalveolar lavage fluid from patients in Wuhan and subsequently confirmed as the cause of COVID 19 by the Chinese center for disease control and prevention (China CDC) (11). Chinese researchers also shared the full genetic sequence of the novel virus through the national institute of health GenBank database (NIHGD) (13) and the global initiation on sharing all influenza data (GISAID) (14).

The spread of this disease from China to other countries have been rapid with 210 countries and territories having confirmed cases. The global count of confirmed cases as of April 17, 2020, 08:56 (GMT) is 2,190,303 with $1,489,604$ active cases and 147, 027deaths

\section{Hosts and Reservoir}

Bats are natural hosts for a large variety of zoonotic viruses with 47 different virus families detected from bat fecal samples in a recent study (15). Generally, it has been thought that Severe Acute Respiratory Syndrome-related coronavirus (SARSr-CoV) -which is mainly found in bats-could cause future disease outbreaks $(16,17)$. Benvenuto and his colleagues found that Bat SARS-like coronavirus was homologous and genetically more similar to the novel coronavirus (2019-nCoV) in comparison all other genomic sequences including the SARS and MERS coronaviruses represented in the phylogenetic tree (18).

Analysis of the amino acid sequence of the seven conserved replicase domains in Open Readind Frame (ORF) 1ab used for CoV species classification showed 94.4\% similarity between the 2019-nCoV and SARSCoV. Thus, suggesting that both viruses belonged to same species, SARSr-CoV. Zhou and his colleagues showed that 2019-nCoV genome was highly similar to Bat coronavirus, BaTCoVRATG13 which was previously detected in Rhinolophus affinis from Yuanan province, with $96.2 \%$ genome sequence identity. The study showed that RATG13 is the closest relative to 2019$\mathrm{nCoV}$ and they form distinct lineage from other SARSrCoVs (11). 
However, coronaviruses which are phylogenetically related to SARS-CoV-2 have been detected in Pangolins (19). High-throughput sequencing of RNA gotten from samples of 18 malayan pangolins (Manis javanica) obtained during anti-smuggling operations performed by Guangxi customs revealed the presence of coronaviruses that fall into the SARS-CoV-2 lineage with similar genomic organization (20). In a study, SARS-CoV-2 exhibits very high sequence similarity to the Guangdong pangolin coronaviruses in the receptor binding domain (RBD; $97.4 \%$ amino acid similarity. The Guangdong pangolin coronavirus and SARS-CoV possess identical amino acids at the five critical residues of the RBD, whereas RaTG13 only shares one amino acid with SARS-CoV-2 having only $89.2 \%$ similarity (20).

Besides the SARS-CoV-2, SARS and MERS have caused serious outbreaks in humans leading to thousands of deaths in the last two decades $(21,22)$. All three coronaviruses have been shown to be of bat origin, SARS and MERS seemed to use different intermediate host $(\mathbf{1 6}, \mathbf{2 3})$. For example, masked palm civet and dromendary camels are the intermediate hosts for SARS CoV (24) and MERS-CoV (25) respectively.Scientists had thought that bat coronaviruses were not generally capable of infecting humans. This observation is consistent with the fact that ACE2 sequence similarity is higher between humans and pangolins (84.8\%) than those between humans and bats (80.8\%-81.4\%; Rhinolophus sp.).

The repeated occurrence of infections with SARSCoV-2 related coronaviruses in Guangxi and Guangdong provinces suggests that this animal may play an important role in the community ecology of coronaviruses (20). However, the role of pangolin as intermediate host has not been proven.

\section{Pathogenesis}

Coronaviruses have different proteins that contribute to its pathogenesis. One of them is the coronavirus spike protein, which is a type I glycoprotein that forms peplomers on the virus. Coronaviruses attach specifically to cellular receptors via the spike protein. The attachment leads to a conformational change of the viral spike protein, and this facilitates fusion of the viral and cellular membranes. The spike protein therefore ensures entry of the virus, cell-to-cell spread of the virus, and it also determines tissue tropism. Coronavirus entry in general, is not said to be $\mathrm{pH}$ dependent. The host range of coronaviruses is very low, as their ability to replicate in a host depends on their ability to interact with its receptors (2).

Another protein responsible for its pathogenesis is the Hemagglutinin-esterase protein, which is peculiar to betacoronaviruses (4). The Hemagglitinin-esterase protein has two domains, a catalytic (esterase) domain and another one that binds to sialic acidcontaining receptors. The binding activity helps in the spread of the virus from cell to cell. The acetylesterase activity becomes important in places like the respiratory tract where it may need to pass through mucus. It also facilitates $S$ (spike) protein-meditated cell entry of the virus $(2,4)$.

The membrane protein is important in viral assembly and virus-host interaction. The nucleocapsid is a structural protein that is involved in transcription and pathogenesis. Mutation of structural proteins could be responsible for the higher ability to infect and enhanced pathogenicity that the bat-like SARS coronavirus (18).

\section{Transmission}

Being a respiratory virus, human-to-human transmission is primarily through respiratory droplets from coughs and sneezes of symptomatic people within a range of 1.8 meters (6ft) (26). Human-tohuman transmission has been confirmed during the SARS-CoV-2 pandemic (27). The SARS-CoV-2 have been found as aerosols floating in air and can cause infection in healthy people (28), however, RNA samples of SARS-CoV-2 have been detected in faecal samples of $2-10 \%$ confirmed patients (29).

The incubation period of the virus, which is the time between exposure of the virus (becoming infected) and symptom onset, is on average of 5-6 days, although it can take up to 14 days (30). During this period, also known as "pre-symptomatic" period, some people can be contagious. Pre-symptomatic transmission has been has been documented through contact tracing and enhanced investigation of clusters of confirmed cases $(30,31)$.

\section{Clinical Manifestation}

Symptoms such as fever, cough, myalgia or fatigue, pneumonia and complicated dyspnea have been reported as the most common symptoms, while, headache, diarrhea, hemoptysis, runny nose and phlegm- producing cough are less commonly reported symptoms $(9,10)$. Patients with severe cases experiences progressive respiratory failure as a result of alveolar damage whereas, patients with mild symptoms have been reported to recover after 1 week (12).

SARS-CoV-2 has been detected in upper and lower respiratory tract, in fecal samples and in blood samples. Laboratory diagnosis is based on a positive reverse transcriptase polymerase chain reaction (RTPCR) test (32).

Radiologic assessments include chest radiography or computed tomography. According to the data 
compiled by Guan and his colleagues, glass-ground opacity was the most common radiologic finding when chest computed tomography (CT) was carried out. No radiographic or CT abnormality was found in 157 of 877 patients (17.9\%) for noncritical cases, and in 5 of 173 patients $(2.9 \%)$ for critical cases. The imaging patterns found on chest CT performed on the case patients were majorly ground-glass opacity (56.4\%) and bilateral patchy shadowing (51.8\%). Other imaging patterns included local patchy shadowing and intestinal abnormalities. Also, lymphocytopenia was found $83.2 \%$ of the patients, thrombocytopenia in $36.2 \%$ and leukopenia in $33.7 \%$ (24). There is however, a reported case of acute necrotizing hemorrhagic encephalopathy associated with COVID19 (33).

\section{Potential Therapeutic Medications for the Treatment Covid-19}

At present, there are no antiviral drugs or vaccines approved to cure or prevent infection by SARS-CoV-2. Owing to this, the best measures to control human coronaviruses remain a strong public health surveillance system and also rapid diagnostic testing and quarantine when necessary. Several studies demonstrate several drugs and the ameliorating effects they proffer, there is no putative prophylactic drug or vaccine proven to prevent infection or cure infection.

\section{Toxilizumab and Siltuximab}

In a study described by Tao and his colleagues (33), most patients with COVID-19 demonstrated higher circulating levels of interleukin-6 (IL-6) after diagnosis. Its mechanism observed to be shared with other cytokine mediated lung injury perpetrated by infectious agents. The use of the IL- 6 inhibitors (monoclonal antibodies), siltuximab and tocilizumab which targets both IL- 6 and IL- 6 receptors may alleviate cytokine-storm related symptoms of COVID19 cases (34) as these have been used to treat cytokine release syndrome following CAR-gaT therapy.

\section{Thalidomide}

In similar case, in Wenzhou city, China, a 45-year-old woman with COVID-19 was treated with thalidomide with low dosage glucocorticoid. In previous outbreaks such as the SARS and MERS corona virus outbreak, high dosage of glucocorticoid was used to suppress cytokine storms. However, it was associated with adverse side effects such as osteoporosis, secondary bacterial infection amongst others. But in low dosage, coupled with thalidomide, pulmonary effusion symptoms and elevated inflammatory cytokines were significantly reduced. In addition, its efficacy is attributed to its ability to activate $T$ cell receptors and
$T$ cells to enhance immune functions, thus ameliorating organ injury by impeding cytokine storms as well as improving the immune system's potential to fight secondary bacterial infection. It also possesses sedative and antiemetic activities that calms anxious patient, thus reducing oxygen consumption, improving digestive symptoms such as vomiting and promotes relief of lung exudation possibly by impeding the growth of new blood vessels (35).

Oseltamivir and abidolhydrochloride, methylprednisolone, moxifloxacin

Other drugs have shown immense potential at ameliorating COVID-19 related symptoms as seen in Wuhan, China, a couple with COVID-19 were treated with methylprednisolone $40 \mathrm{mg}$ (once), human gamma globulin $10 \mathrm{~g}$ changed later on to $5 \mathrm{~g}$. Also, drugs used to treat atypical pathogens such as moxifloxacin for mycoplasma and chlamydia, oseltamivir and abidolhydrochloride for influenza virus and a Chinese patent medicine Tanreqing was administered for adjunctive therapy. The patients showed normal condition after being re-examined for all indication of inflammation. These medications were administered before retrospective studies showed positive results to COVID-19 caused by SARS-CoV-2. Since the patients exhibited no underlying health conditions, recovery could also be attributed to a competent immune system. Nevertheless, these holds promise in alleviating COVID-19 related inflammations (36).

\section{Lopinavir/Ritonavir}

In another study, Lim and his colleagues (37) reported lopinavir/ritonavir to be successful in the treatment of 54-year old patient with COVID-19. This was administered day 10 of illness; 2 tablets (lopinavir $200 \mathrm{mg}$, ritonavir $50 \mathrm{mg}$ ). Clinical results show a decrease in $\beta$-coronavirus load with no detectable titre since it was administered. However, speculations pose that improved conditions could be a synergistic effect of natural healing process and the medications or either. Further experiments would be necessary to establish reproducible effects of these medications.

\section{Chloroquine and Hydroxychloroquinone}

Chloroquine and hydroxychloroquinone were reported to be effective at preventing escalation of COVID-19 associated pneumonia. Clinical trial showed that chloroquine could block COVID-19 infection at low micromolar $(\mu \mathrm{M})$ concentrations with half maximal effective concentration of $1.13 \mu \mathrm{M}$ and a half cytotoxic concentration greater than $100 \mu \mathrm{M}$. In addition, it has also been proven to lung imaging findings, promote a virus negative conversion and shorten the disease course. Its antiviral activity is attributed to its potential to increase endosomal $\mathrm{pH}$ 
required for virus/cell infusion as well as interfere with glycosylation of cellular receptors of SARS-CoV. In addition to its antiviral properties, it also has antiinflammatory properties which render it a suitable choice for COVID-19 related pneumonia (38).

\section{Blood Serum}

In previous outbreaks known to have ravaged the world, blood plasma from convalescents have been proven to be antidotalin the treatment of Severe Acute Respiratory Syndrome (SARS) and Ebola has shown significant success. This treatment method dates back to the 1918 H1N1 influenza virus where more than 1700 people received blood serum survivors. In 2002-2003, during the SARS outbreak, a study was carried out on 80 persons in Hongkong with convalescent serum. Clinical reports revealed that people treated within two weeks of showing symptoms had a higher chance of recuperating and being discharged from the hospital than those who weren't treated. Likewise blood sera from Ebola survivors have been tested on at least two outbreaks of Ebola in Africa with measurable success (39). Test trials using convalescent serum plasma to treat 13 people critically ill with COVID-19 virus in China, revealed the cessation of circulation of the virus within the patients. Though their conditions continued to deteriorate indicating disease progression too far along for the therapy to be effective (Liang $\mathrm{Yu}$, Zhejiang University School of Medicine in China). In spite of this, this treatment holds great potential to abate spread of infection to forestall immense mortality that could arise from delay in the manufacture of a suitable drug/vaccine.

\section{Remdesivir}

Remdesivir is a prodrug of a nucleoside analogue which is metabolized intracellularly to adenosine triphosphate which impedes viral RNA polymerase activity, thus hindering translation of viral genetic material. It has broad spectrum activity against notable viruses which includes filoviruses such as Ebola and corona viruses (Severe Acute respiratory Syndrome Coronavirus (SARS-CoV) and Middle East Respiratory Syndrome Coronavirus (MERS-CoV)). Studies have shown that it possesses some activity against SARS CoV-2 causative agent of COVID-19 illness. More so, remdesivir has shown to prophylactic and therapeutic efficacy in non-clinical models of these coronaviruses (40).

The data of the study showed that gender, region of enrollment, coexisting condition and duration of symptoms before the treatment, was not very significant in measurement of clinical improvement of the $68 \%$ of patients that showed improvement with the treatment, $13 \%$ including $18 \%$ of patients on invasive ventilation, died after completion of remdesivir treatment.

Although shown to be somewhat effective, the safety of this medication has not yet been ensured as $60 \%$ of patients exposed to this treatment reported adverse effects in the follow up (40). The most frequent includes: increased hepatic enzymes, diarrhea, rash, renal impairment and hypotension. In some cases, severe adverse effects were reported. Most common includes: multiple organ-dysfunction syndrome, septic shock, acute kidney injury, and hypotension. These adverse effects were more common in patients receiving invasive ventilation.

\section{Conclusion}

Several studies have demonstrated the use of certain drugs to combat infection as some of which target impediment of cytokine-storms which contributes to inflammation (toxilizumab, siltuximab, thalidomide in addition to its sedative, antiemetic and lung exudation relief properties), viral RNA polymerase activity that function in translation of genetic material (remdesivir) and interfere with glycosylation of cellular receptors of SARS-CoV (chloroquinone and hydroxychloroquinone). Phylogenetic studies suggest the novel virus to be closely related to the BaTCoVRATG13 virus and Guangdong pangolin coronavirus and predicted the SARSr-CoV to be of potential harm in the near future. Since bats have been known to be the main reservoir for coronaviruses that have plagued the world, a recurrent surge of another coronavirus of zoonotic origin might occur. Perhaps early identification and characterization of coronaviruses peculiar to bats and pangolins might be a potential solution to the dilemma of drug/vaccine synthesis designed to combat future infections. A major harbinger to the development of novel coronavirus-related infections is the unpredictable wave of genetic mutations supported by natural selection which have been known to confer greater virulence to this group of viruses, bridging the inter-specie barrier between humans and bats. The novel coronavirus SARS-CoV-2, although known to be lethal has been reported to be combatable.

\section{Acknowledgment}

We are grateful to all the experts who have given insights for the writing of this paper. The paper was financed by all participant authors. 


\section{Authors Contributions}

Ekpunobi designed the study, Ekpunobi, Markjonathan, Olanrewaju and Olanihun wrote the manuscript and participated in literature review. All authors contributed equally, read and approved the final manuscript

\section{Conflict of Interest}

Authors declared no conflict of interests.

\section{References}

1. WHO. Coronavirus. [Cited 2020 Apr 12]. Available from: https://www.who.int/healthtopics/coronavirus.

2. Weiss SR, Navas-Martin S. Coronavirus pathogenesis and the emerging pathogen severe acute respiratory syndrome coronavirus. Microbiol Mol Biol Rev. 2005; 69: 635-664 [DOI:10.1128/MMBR.69.4.635664.2005] [PMID] [PMCID]

3. Hussain S, Pan J, Chen Y, Yang Y, Xu J, Peng Y, et al. Identification of novel subgenomic RNAs and noncanonical transcription initiation signals of severe acute respiratory syndrome coronavirus. J Virol. 2005; 79(9): 5288-5295. [DOI:10.1128/JVI.79.9.5288-5295.2005] [PMID] [PMCID]

4. Lissenberg A, Vrolijk MM, van Vliet ALW, Langereis MA, de Groot-Mijnes JDF, Rottier PJM, et al. Luxury at a Cost? Recombinant Mouse Hepatitis Viruses Expressing the Accessory Hemagglutinin Esterase Protein Display Reduced Fitness In Vitro. J Virol. 2005; $\quad$ 79(24): 15054-15063. [DOI:10.1128/JVI.79.24.15054-15063.2005] [PMID] [PMCID]

5. Su S, Wong G, Shi W, Liu J, Lai ACK, Zhou J, et al.Epidemiology, genetic recombination, and pathogenesis of coronaviruses. Trends Microbiol. 2016; 24: 490-502 [DOI:10.1016/i.tim.2016.03.003] [PMID] [PMCID]

6. Zhu N, Zhang D, Wang W, Li X, Yang B, Song J, et al. A novel coronavirus from patients with pneumonia in China, 2019. N Engl J Med. 2020; 382:727-733 [DOI:10.1056/NEJMoa2001017] [PMID] [PMCID]

7. Lu R, Zhao X, Li J, Niu P, Yang B, Wu H, et al. Genomic characterisation and epidemiology of 2019 novel coronavirus: implications for virus origins and receptor binding. Lancet. 2020; 395(10222): 391393. [DOI:10.1016/S0140-6736(20)30251-8]

8. Gorbalenya AE, Baker SC, Baric RS, de Groot RJ, Drosten C, Gulyaeva AA, et al."The species Severe acute respiratory syndrome-related coronavirus: classifying 2019-nCoV and naming it SARS-CoV-2". Nature Microbiology. 2020; 5 (4): 536-544. [DOI:10.1038/s41564-020-0695-z] [PMID] [PMCID]
9. Huang C, Wan, Y, Li X, Ren L, Zhao J, Hu Y, et al. Clinical features of patients infected with 2019 novel coronavirus in Wuhan, China. Lancet. 2020; 395:497506. [DOI:10.1016/S0140-6736(20)30183-5]

10. CDC. Novel coronavirus, Wuhan, China. [Cited 2020 Apr 14] Availabe from; https://www.cdc.gov/ coronavirus/2019-nCoV/summary.html.

11. Zhou $P$, Yang $X L$, Wang $X G$, Hu B, Zhang L, Zhang $W$, et al."A pneumonia outbreak associated with a new coronavirus of probable bat origin". Nature. 2020; 579 (7798): 270-273 [DOI:10.1038/s41586-0202012-7] [PMID] [PMCID]

12. Li Q, Guan $X$, Wu $P$, Wang $X$, Zhou $L$, Tong $Y$, et al. Early Transmission Dynamics in Wuhan, China, of Novel Coronavirus-Infected Pneumonia. N Engl J Med. 2020; 382:1199-1207 [DOI:10.1056/NEJMoa2001316] [PMID] [PMCID]

13. National Institutes of Health. GenBank overview (https://www.ncbi.nlm.nih.gov/genbank/).

14. GISAID (Global Initiative on Sharing All Influenza Data) home page (https://www.gisaid.org/)

15. Mendenhall IH, Wen DLH, Jayakumar J, Gunalan V, Wang L, Mauer-Stroh S, et al. Diversity and Evolution of Viral Pathogen Community in Cave Nectar Bats (Eonycteris spelaea). Viruses 2019; 11: 250. [DOI:10.3390/v11030250] [PMID] [PMCID]

16. Cui J, Li F, Shi ZL. Origin and evolution of pathogenic coronaviruses. Nat Rev Microbiol. 2019; 17:181-192. [DOI:10.1038/s41579-018-0118-9] [PMID] [PMCID]

17. Fan $Y$, Zhao K, Shi ZL, Zhou P. Bat coronaviruses in China. Viruses 2019; 11: 210 [DOI:10.3390/v11030210] [PMID] [PMCID]

18. Benvenuto D, Giovanetti M, Ciccozzi A, Spoto S, Angeletti S, Ciccozzi M. The 2019-new coronavirus epidemic: Evidence for virus evolution. Jour Med Viro. 2020; 92(4): 1-5. [DOI:10.1002/jmv.25688] [PMID] [PMCID]

19. Cyranoski D. Mystery deepens over animal source of coronavirus. Nature. 2020; 579(7797): 18-19. [DOI:10.1038/d41586-020-00548-w] [PMID]

20. Lam TT, Shum MH, Zhu H, Ni X, Liao Y, Wei W, et al. (2020). Identifying SARS-CoV-2 related coronaviruses in Malayan pangolins. [Published online ahead of print, 202 Mar 26]. Nature. [DOI:10.1038/s41586-020-2169-0] [PMID]

21. Zaki AM, Van Boheemen S, Bestebroer TM, Osterhaus AD, Fouchier RA. Isolation of a novel coronavirus from a man with pneumonia in Saudi Arabia. N Engl Jour Med. 2012; 367(19):1814-1820. [DOI:10.1056/NEJMoa1211721] [PMID]

22. Cunha CB, Opal SM. Middle East respiratory syndrome (MERS): A new zoonotic viral pneumonia. Virulence. 2014; 5(6):650-654. [DOI:10.4161/viru.32077] [PMID] [PMCID] 
23. Hu B, Ge X, Wang L. Shi Z. Bat origin of human coronaviruses. Virol J 2015; 12: 221 [DOI:10.1186/s12985-015-0422-1] [PMID] [PMCID]

24. Guan Y, Zheng BJ, He YQ, Liu XL, Zhuang ZX, Cheung $\mathrm{CL}$, et al. Isolation and characterization of viruses related to the SARS coronavirus from animals in southern China. Science. 2003; 302: 276-278. [DOI:10.1126/science.1087139] [PMID]

25. Alagaili AN, Briese T, Mishra N, Kapoor V, Sameroff SC, Burbelo PD, et al. Middle East respiratory syndrome coronavirus infection in dromedary camels in Saudi Arabia. mBio. 2014; 5: e00884-14. [DOI:10.1128/mBio.01002-14] [PMCID]

26. Centers for Disease Control and Prevention (CDC). [Cited 2020 Apr 13]. "How COVID-19 Spreads".

27. Chan JF, Yuan S, Kok KH, To KK, Chu H, Yang J, et al. "A familial cluster of pneumonia associated with the 2019 novel coronavirus indicating person-to-person transmission: a study of a family cluster". Lancet 2020; 395(10223): 514-523. [DOI:10.1016/S01406736(20)30154-9]

28. WHO Director-General's opening remarks at the media brieng on COVID-19 -3 March 2020. [Cited 2020 Apr 13] Available from; https://www.who.int/dg/speeches/detail/whodirectorgeneral-s-opening-remarks-at-the-mediabrieng-oncovid-19-3-march-2020

29. Gao QY, Chen YX, Fang JY. 2019 novel coronavirus infection and gastrointestinal tract. J dig dis. 2020; 21(3): 125-126 [DOI:10.1111/1751-2980.12851] [PMID] [PMCID]

30. World Health Organization. Report of the WHOChina Joint Mission on Coronavirus Disease 2019 (COVID-19) 16-24 February 2020 [Internet]. Geneva: World Health Organization; 2020 Available from: https://www.who.int/docs/defaultsource/coronaviruse/who-china-joint-mission-oncovid-19-finalreport.pdf

31. Wei WE, Li Z, Chiew CJ, Yong SE. Presymptomatic Transmission of SARS-CoV-2 - Singapore, January 23 March 16, 2020. MMWR. 2020; 69(14): 411-415 [DOI:10.15585/mmwr.mm6914e1] [PMID] [PMCID]

32. WHO. Laboratory testing of 2019 novel coronavirus (2019-nCoV) in suspected human cases: interim guidance. [Cited 2020 Apr 13]. Available from; 2 March 2020. No. WHO/COVID19/laboratory/2020.4. World Health Organization, 2020.

33. Poyiadji N, Shahin G, Noujaim D, Stone M, Patel S, Griffith B. COVID-19-associated acute hemorrhagic necrotizing encephalopathy: CT and MRI features. Radiology 2020; 201187 [DOI:10.1148/radiol.2020201187] [PMID] [PMCID]

34. Liu T, Zhang J, Yang Y, Zhang L, Ma H, Li Z, et al."The potential role of IL- 6 in monitoring coronavirus disease 2019". J Autoimmun. 2020; 10: 102452 [DOI:10.1016/j.jaut.2020.102452] [PMID] [PMCID]

35. Chen C, Qi F, Shi K, Li Y, Li J, Chen Y, et al. "Thalidomide Combined with Low-dose Glucocorticoid in the Treatment of COVID-19 Pneumonia". Preprints 2020; 2020020395 [DOI:10.1002/ctm2.35]

36. Zhang Z, Li X, Zhang W, Shi U, Zheng Z, Wang T. "Clinical features and Treatment of 2019-nCoV pneumonia patients in Wuhan: Report of a couple cases" Virol Sin. 2020; 1-7 [DOI:10.1007/s12250-02000203-8] [PMID] [PMCID]

37. Lim J, Jeon $S$, Shin $H$, Kim $M$, Seong $Y$, Lee $W$, et al. "Case of the Index Patient Who Caused Tertiary Transmission of Coronavirus Disease 2019 in Korea: the Application of Lopinavir/Ritonavir for the Treatment of COVID-19 Pneumonia Monitored by Quantitative RT-PCR" J Korean Med Sci. 2020; 35(6):e79 [DOI:10.3346/jkms.2020.35.e79] [PMID] [PMCID]

38. Gao J, Tian Z, Yang X. "Breakthrough: Chloroquine phosphate has shown apparent efficacy in treatment of COVID-19 associated pneumonia in clinical studies". BioSci Trends.2020; 14(1):72-73. [DOI:10.5582/bst.2020.01047] [PMID]

39. Maxmen A. "How blood from coronavirus survivors might save lives" Nature. 2020; 580:16-17 [DOI:10.1038/d41586-020-00895-8] [PMID]

40. Grein J, Ohmagari N, Shin D, Diaz G, Asperges E, Castagna A, Feldt T. et al. "Compassionate Use of Remdesivir for Patients with Severe Covid-19". N Engl J Med. 2020 Apr 10; [epub]. 